\title{
Spotting Significant Changing Subgraphs in Evolving Graphs
}

\author{
Zheng Liu, Jeffrey Xu Yu, Yiping Ke \\ The Chinese University of Hong Kong \\ \{zliu,yu,ypke\}@se.cuhk.edu.hk
}

\author{
Xuemin Lin \\ The University of New South Wales \\ 1xue@cse.unsw.edu.au
}

\author{
Lei Chen \\ Hong Kong University of Science and Technology \\ leichen@cs.ust.hk
}

\begin{abstract}
Graphs are popularly used to model structural relationships between objects. In many application domains such as social networks, sensor networks and telecommunication, graphs evolve over time. In this paper, we study a new problem of discovering the subgraphs that exhibit significant changes in evolving graphs. This problem is challenging since it is hard to define changing regions that are closely related to the actual changes (i.e., additions/deletions of edges/nodes) in graphs. We formalize the problem, and design an efficient algorithm that is able to identify the changing subgraphs incrementally. Our experimental results on real datasets show that our solution is very efficient and the resultant subgraphs are of high quality.
\end{abstract}

\section{Introduction}

Discovering patterns in graph data is a challenging task with many applications in Web analysis, social networks, telecommunication, sensor networks, etc. Due to the expressive power of graphs, graph patterns have to represent complex structural relationships among objects in various domains in the real world. In the literature, the existing work $[5,4,7,9]$ discovers complex relationships in a large graph that is static.

However, many applications show that graphs are evolving over time. In social science, large social networks are changing, and social network changes are caused by the proximity changes [9]. In bioinformatics, finding coevolution relationships of structure and function in the structural genomic is an important task to understand evolution progresses [8]. In network, traffic jam occurs at one link may affect the traffic routing in a large range. Monitoring the dynamic topology changes and their influences provides with network administrators the insights on network configuration [3]. Also, in wireless sensor networks, query processing is done by exchanging information between sensors where the communication range of a sensor is limited. The fact that some sensor runs out of power has impacts on the other sensors in terms of network routing and therefore query processing time. In a sensor network, it is important to note, even beforehand, which subgraphs will be affected significantly when such a change occurs.

Such changes can be modeled in a sequence of large graphs, $G=\left(G_{1}, G_{2}, \cdots\right)$, where nodes/edges can be added and/or deleted into/from $G_{i}$ which results in another large graph $G_{i+1}$, and $G$ is considered as an evolving graph. In this work, we take an edge-centric view regarding changes. We focus on edge changes (deletion/addition) which will cause structural changes. On the other hand, vertex changes also have impacts on structural changes. But adding isolated vertices before they are connected to any other vertices seems less important, while deleting vertices can be considered as removing edges connected to the deleted vertices.

Given two graphs $G_{i}$ and $G_{i+1}$ at time $t_{i}$ and time $t_{i+1}$, there are many small subgraphs that change while the majority of the graph remains unchanged. A small changing subgraph can be a connected subgraph where every edge is changed (deleted from $G_{i}$ or added into $G_{i+1}$ ), and such a small changing subgraph can be easily identified. However, the influence of a single edge change (deletion/addition) on the other parts of the large graph is more important than the physical change itself. For example, when a researcher $A$ works with another researcher $B$ for a new research issue, $A$ 's collaborators and $B$ 's collaborators may have new opportunities to work together. Consider the two researchers as two vertices. The newly added edge between them may change the closeness of the vertices that are directly/indirectly connected to the two vertices. Suppose that the closeness of two vertices can be measured. A changing subgraph is an induced subgraph in which the closeness be- 


\begin{tabular}{l|l}
\hline Symbol & Definition \\
\hline \hline$G$ & An evolving graph \\
$G_{i}$ & The snapshot of evolving graph $G$ at time $t_{i}$ \\
$A_{i}$ & The adjacency matrix of graph $G_{i}$ \\
$P_{i}$ & The transition matrix of graph $G_{i}$ \\
$v_{j}$ & A vertex on a graph \\
$N\left(v_{j}\right)$ & The set of neighbors of vertex $v_{j}$ \\
$d(j)$ & The sum of edge weights between vertex $v_{j}$ and $N\left(v_{j}\right)$ \\
$D_{i}$ & The diagonal matrix where $d_{j j}=d\left(v_{j}\right)$ at time $t_{i}$ \\
$\Pi_{i}$ & The vertex closeness matrix at time $t_{i}$ \\
$V I_{i}$ & The vertex importance score at time $t_{i}$ \\
\hline
\end{tabular}

Table 1. Notations

tween vertices changes. In this paper, we focus on the problem of spotting significant changing induced subgraphs in an evolving graph. The issues that we concentrate on include how to measure the closeness changes between two vertices that are caused by some edge changes, how to identify the boundary of the influences of a change, and how to determine a changing subgraph in which changing parts have influences on each other.

The main contributions of this paper are summarized below.

- We formalize the problem of spotting significant changing subgraphs in an evolving graph and propose to measure the vertex closeness with structure information using neighborhood random walks.

- We develop an incremental algorithm to speed up the vertex closeness computation, as well as a novel strategy about expanding the important vertex to acquire the connected induced subgraph which can reflect the closeness change between vertices.

- We present an evaluation of our proposed approach by using various large real data sets demonstrating that our method is able to find the suitable subgraph effectively and efficiently.

\section{Changing Subgraph Discovery}

We define an evolving graph as a sequence of undirected graphs, denoted as $G=\left(G_{1}, G_{2}, \cdots\right)$, where $G_{i}\left(V_{i}, E_{i}\right)$ is a snapshot of graph $G$ at time $t_{i}$ with a set of vertices $V_{i}$ and a set of edges $E_{i}$. For simplicity, given two graphs $G_{i}\left(V_{i}, E_{i}\right)$ and $G_{i-1}\left(V_{i-1}, E_{i-1}\right)$, the two sets of vertices, $V_{i}$ and $V_{i-1}$, are identical, while the two sets of edges, $E_{i}$ and $E_{i-1}$, are possibly different. The notations used in this paper are summarized in Table 1.

Consider an evolving graph. An edge change may make some vertices become closer and at the same time may make some other vertices become looser. As shown in Figure 1, there are two graphs $G_{1}$ and $G_{2}$ in an evolving graph at time $t_{1}$ and $t_{2}$, respectively. At time $t_{1}$, there is an edge between

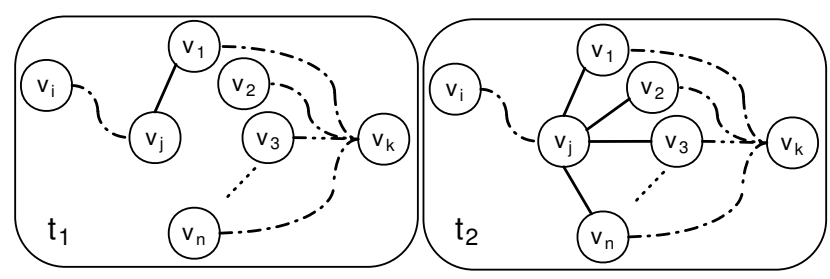

Figure 1. Changes

$v_{i}$ and $v_{j}$, and there is a path between $v_{j}$ and $v_{k}$. At time $t_{2}$, there are more paths from $v_{j}$ to $v_{k}$, where the edge between $v_{i}$ and $v_{j}$ remains unchanged. In comparison with $G_{1}$ at time $t_{1}$, the closeness between $v_{j}$ and $v_{k}$ becomes closer, because the newly added edges make it easier for $v_{j}$ to traverse to $v_{k}$. On the other hand, the closeness between $v_{j}$ and $v_{i}$ becomes looser, because $v_{j}$ has more opportunities to traverse to other vertices. This fact motivates us to consider an accumulative score that measures the overall impacts of all changes on a vertex when a graph evolves. Reconsidering $v_{j}$ in Figure 1, we need to consider the relationships between $v_{j}$ and $v_{i}$, between $v_{j}$ and $v_{k}$, and between $v_{j}$ and any other vertex, in order to judge the change influence on $v_{j}$. Given such an accumulative score, it becomes possible to find significant changing subgraphs when a graph evolves.

In this paper, we explore this issue using neighborhood random walks on graphs to help spotting significant changing subgraphs. We first review some basic concepts of random walks on graphs. Let $A_{i}$ denote the adjacency matrix of a weighted graph $G_{i}$, where $A_{i}(j, k)$ maintains the weight for the edge $\left(v_{j}, v_{k}\right)$. A random walk on $G_{i}$ is performed in the following way. A particle starts at a certain vertex $v_{0}$, which is a vertex involved in an edge change. Suppose it walks to a vertex $v_{s}$ in the $s$-th step and it is about to move to one of the neighbors of $v_{s}$, denoted as $v_{t} \in N\left(v_{s}\right)$, with probability $p_{s t}$, where $p_{s t}$ is $A_{i}(s, t) / \sum_{v_{k} \in N\left(v_{s}\right)} A_{i}(s, k)$, and $N\left(v_{s}\right)$ contains all neighbors of vertex $v_{s}$. The vertex sequence of the random walk is a Markov chain. Let $D_{i}$ be the diagonal matrix with the diagonal value $d(s)=\sum_{v_{k} \in N\left(v_{s}\right)} A_{i}(s, k)$, then the transition probability matrix $P_{i}$ of the Markov chain for graph $G_{i}$ is

$$
P_{i}=D_{i}^{-1} A_{i} .
$$

The probability of going from $v_{j}$ to $v_{k}$ through a random walk of length $l$ can be obtained by multiplying the transition probability matrix $l$ times and is given as $P_{i}^{l}(j, k)$.

A fixed step approach with restart (fixed $l$ and $0<c<$ 1 ): With a fixed $l$, we focus on the local structural information using neighbors of a vertex, $v_{j}$, from which the random walk starts. The vertex, $v_{j}$, to start random walks is the vertex that is involved in an edge change. The neighbors of $v_{j}$ are the vertices that $v_{j}$ can reach in $l$ steps. Random walks are only conducted in the $l$-step neighborhood of the vertex $v_{j}$ with a restart probability $c$. It is important to note that our 
algorithm is designed in a way that a user can enlarge the $l$ value if needed at run time. We adopt the similar expected $f$-distance in $[6,5]$. In the expected $f$-distance, a parameter $c$ is used. We prove that such a parameter $c$ is the restart probability used in [7] with minor difference which can be ignored. Due to the space limit, we omit the proof. In short, neighborhood random walk distance, which is also called the vertex closeness, is the expected $f$-distance defined on random walks whose length is smaller or equal to $l$.

Definition 1 Neighborhood Random Walk Distance (Vertex Closeness): Let $P_{i}$ be the $n \times n$ transition probability matrix of a graph $G_{i}$. Given $l$ as the length that a random walk can go, the neighborhood random walk distance $\Pi^{l}(j, k)$ from $v_{j}$ to $v_{k}$ is defined as follows:

$$
\pi(j, k)=\sum_{\tau: v_{j} \rightsquigarrow v_{k} ; \text { length }(\tau) \leq l} p(\tau) c(1-c)^{l e n g t h(\tau)},
$$

where $0<c<1$, and $\tau$ is a path from $v_{j}$ to $v_{k}$ whose length is length $(\tau)$ with transition probability $p(\tau)$.

The matrix form of the neighborhood random walk distance is as follows.

$$
\Pi_{i}^{l}=\sum_{\gamma=1}^{l} c(1-c)^{\gamma} P_{i}^{\gamma} .
$$

Here, $P_{i}$ is the transition probability matrix for graph $G_{i}$, and $\Pi_{i}$ is the neighborhood random walk distance matrix for graph $G_{i}$. We then define the importance score of a vertex as the accumulative change of its closeness to other vertices in Eq. (3).

$$
V I_{i}\left(v_{j}\right)=\sum_{v_{k} \in V_{i}}\left|\Pi_{i-1}^{l}(j, k)-\Pi_{i}^{l}(j, k)\right| .
$$

Here, $V I_{i}\left(v_{j}\right)$ is the importance score of a vertex $v_{j}$ when a graph evolves from graph $G_{i-1}$ to $G_{i}$.

We propose a two-step framework to spot significant changing subgraphs in an evolving graph. First, we compute the importance score $V I_{i}\left(v_{j}\right)$ for any vertex $v_{j}$ in graph $G_{i}$ that is involved in edge changes. Second, based on the importance scores, we find significant changing subgraphs.

\section{Vertex Importance Score Computation}

In this section, we discuss in detail how to calculate the difference of vertex closeness in two graphs $G_{i-1}$ and $G_{i}$ and the vertex importance scores.

\subsection{The Straightforward Algorithm}

We can develop a straightforward algorithm to compute the difference of vertex closeness and the vertex importance score based on the definitions. The straightforward algorithm iteratively calculates the respective closeness matrices $\Pi_{i-1}$ and $\Pi_{i}$ at time $t_{i-1}$ and $t_{i}$ based on Eq. (2) by the power method. The closeness difference matrix is simply computed as $C_{i}=\Pi_{i}-\Pi_{i-1}$, based on which the importance scores of vertices can be easily computed by Eq. (3). The time complexity of the straightforward algorithm is $O\left(l^{3}\right)$. One can use the fast sparse matrix multiplication instead of the normal matrix multiplication to improve the speed, but usually that is not enough to lower the running time especially when $G$ is large and there are a lot of edge changes.

\subsection{A Novel Incremental Algorithm}

In this section, we introduce a novel incremental algorithm that computes the closeness difference directly for those vertex pairs with changing closeness.

Let us start from a simple case. Suppose that there is only one edge $e$ that is different between two graphs $G_{i}$ and $G_{i-1}$. It can be either the addition of $e$ to $G_{i}$ or the deletion of $e$ from $G_{i-1}$. The question is to identify those vertex pairs whose closenesses change due to the difference of $e$, as well as the quantities changed. Recall our closeness measure in Eq. (1). The answer to the above question is that if a vertex pair has at least one tour path passing through the edge $e$ or one of the two vertices incident to edge $e$, the closeness of the vertex pair changes. By identifying those paths, we can find the vertex pairs with changing closenesses. Furthermore, the summation of the probability of these paths is exactly the quantity changed in the closeness of each vertex pair.

By Eq. (2), the iterative form of the vertex closeness is

$$
\Pi_{i}^{l}=\sum_{\gamma=1}^{l} c(1-c)^{\gamma} P_{i}^{\gamma}=c(1-c)^{l} P_{i}^{l}+\Pi_{i}^{l-1} .
$$

Therefore, the closeness difference matrix is

$$
\Delta \Pi_{i}^{l}=c(1-c)^{l}\left(P_{i}^{l}-P_{i-1}^{l}\right)+\Delta \Pi_{i}^{l-1} .
$$

By Eq. (5), we can see that the key step in computing $\Delta \Pi_{i}^{l}$ is to compute $\left(P_{i}^{l}-P_{i-1}^{l}\right)$, which is easy when $l=1$. When $l>1$, obviously we cannot compute it in a naive way by the power method since it is computational expensive. Recall that $P_{i}^{l}(j, k)$ is the probability of going from $v_{j}$ to $v_{k}$ through random walks of length $l$ on graph $G_{i}$. We now show how to calculate $\Delta P_{i}^{l}=P_{i}^{l}-P_{i-1}^{l}$ in an efficient way. Apparently,

$$
P_{i}^{l}(j, k)=\sum_{\tau: v_{j} \rightsquigarrow v_{k}} p_{i}(\tau)
$$

where $\tau$ is a tour path from vertex $v_{j}$ to $v_{k}$, and $p_{i}(\tau)$ is the probability of path $\tau$ in $G_{i}$. Suppose $\tau=$ 
$\left\langle v_{1}, v_{2}, \ldots, v_{l}\right\rangle$, where $v_{1}=v_{j}$ and $v_{l}=v_{k}$, then $p_{i}(\tau)=$ $\prod_{m=1}^{l-1} A_{i}\left(v_{m}, v_{m+1}\right) / d\left(v_{m}\right)$. The sum of the probability of all these distinct tour paths is $P_{i}^{l}(j, k)$.

In order to compute $\Delta P_{i}^{l}$, we only need to consider the different paths on $G_{i}$ and $G_{i-1}$, as well as the difference in the probability of the same paths. For simplicity, we only discuss the case when there are only additions of edges or increase of edge weights. We will show later that our algorithm can handle deletions of edges and decrease of edge weights as well.

Let $\left(v_{m}, v_{n}\right)$ be one of the added edges or one of the edges whose weights increase. For any vertex pair $\left\{v_{j}, v_{k}\right\}$, if there is a tour path $\tau$ of the maximum length $l$ starting from $v_{j}$, passing through the edge $\left(v_{m}, v_{n}\right)$ and ending at $v_{k}$, then the vertex closeness $\Pi_{i}(j, k)$ will increase by $p_{i}(\tau)$, since this path does not exist in $G_{i-1}$. On the other hand, if there is a tour path $\tau$ of the maximum length $l$ starting from $v_{j}$, passing $v_{m}$ or $v_{n}$ or both, and ending at $v_{n}$, but without passing through $\left(v_{m}, v_{n}\right)$, then the vertex closeness $\Pi_{i}(j, k)$ will decrease by $\left(p_{i-1}(\tau)-p_{i}(\tau)\right)$, since the path $\tau$ exists in both $G_{i}$ and $G_{i-1}$, and with the increase of $d(m)$ and $d(n)$ in $G_{i}$, the probability of the path $\tau$ decreases. We formalize the above analysis in Theorem 3.1.

Theorem 3.1 Given two graphs $G_{i}$ and $G_{i-1}$ of an evolving graph $G$, let $\left(v_{m}, v_{n}\right)$ denote the changing edge, then $\Delta P_{i}^{l}(j, k)$ can be computed as follows:

$$
\Delta P_{i}^{l}(j, k)=\sum_{\tau: v_{j} \rightsquigarrow v_{k} ;\left(v_{m}, v_{n}\right) \in \tau} p(\tau)+\sum_{\tau: v_{j} \rightsquigarrow v_{k} ;\left(v_{m}, v_{n}\right) \notin \tau ; v_{m} \text { or } v_{n} \in \tau}\left(p_{i}(\tau)-p_{i-1}(\tau)\right) .
$$

Theorem 3.1 suggests an effective way to calculate the change quantity of the closeness between vertex pairs. The key is to find all the related paths distinctly and completely so that the change quantity is computed correctly. To enumerate all the possible positions of the edge $\left(v_{m}, v_{n}\right)$ in a path $\tau$ is obviously not a good solution due to the exponential number of combinations with respect to the number of changing edges and the range $l$.

We first discuss the case of the path $\tau: v_{j} \rightsquigarrow v_{k}$ when $\left(v_{m}, v_{n}\right) \in \tau$. We can calculate the closeness difference in the following way. For the changing edge $e=\left(v_{m}, v_{n}\right)$, we first calculate the probability of a path $\tau_{1}$ from $v_{j}$ to $v_{m}$ with length $l_{1}$, where $l_{1} \leq l-1$. We then calculate the probability of a path $\tau_{2}$ from $v_{n}$ to $v_{k}$ with length $l_{2}=l-l_{1}$. In this way, we ensure that the computed paths from $v_{j}$ to $v_{k}$ passing the edge $\left(v_{m}, v_{n}\right)$ is of length $l$. The closeness difference that is accounted for such paths can be computed as

$$
\pi_{1}(j, k)=\sum_{\tau_{1}: v_{j} \rightsquigarrow v_{m} ; \tau_{2}: v_{n} \rightsquigarrow v_{k}} p\left(\tau_{1}\right) P_{i}(m, n) p\left(\tau_{2}\right),
$$

where $\pi_{1}(j, k)$ denotes the first term of $\Delta P_{i}^{l}(j, k)$ in Eq. (7).

In order to compute $p\left(\tau_{1}: v_{j} \rightsquigarrow v_{m}\right)$ and $p\left(\tau_{2}: v_{n} \rightsquigarrow\right.$ $v_{k}$ ) correctly without missing and double-computing any path, we do not allow a path $\tau_{1}$ from $v_{j}$ to $v_{m}$ to pass $e$, while we do not have this restriction on path $\tau_{2}$.

As for the other case of the path $\tau: v_{j} \rightsquigarrow v_{k}$ when $v_{m}$ or $v_{n} \in \tau$ but $\left(v_{m}, v_{n}\right) \notin \tau$, the closeness difference can be computed in a similar way:

$$
\pi_{2}(j, k)=\sum_{\substack{\tau_{3}: v_{j} \rightsquigarrow v_{m} ; \tau_{4}: v_{m} \rightsquigarrow v_{k} \\ o r \tau_{3}: v_{j} \rightsquigarrow v_{n} ; \tau_{4}: v_{n} \rightsquigarrow v_{k}}} p\left(\tau_{3}\right) p\left(\tau_{4}\right),
$$

where $\pi_{2}(j, k)$ denotes the second term of $\Delta P_{i}^{l}(j, k)$ in Eq. (7). For the correctness of computation, we do not allow $\tau_{3}$ to contain the vertex $v_{m}$ (or $v_{n}$ when $\tau_{3}$ is from $v_{j}$ to $v_{n}$ ) and we do not allow $\tau_{4}$ to contain the edge $e$.

We handle all the changed vertices together in matrix form instead of one by one. We use six arrays to store the corresponding probability of the four types of paths $\tau_{1}$ to $\tau_{4}$ discussed above, the total number of whose entries is much small than $n^{2}$.

We have discussed how to handle the additions of edges and the increase of edge weights. In fact, our algorithm can also handle the situation when there are deletions of edges and decrease of edge weights. Let us first suppose that there are only deletions of edges and decrease of edge weights from $G_{i-1}$ to $G_{i}$. It is easy to see that this is exactly the same as the evolvement from $G_{i}$ to $G_{i-1}$, where only additions of edges and increase of edge weights happen. The only difference is that the closeness difference matrix should be multiplied by -1 . In general, we can first handle all the additions of edges, together with the increase of edge weights, and then handle the deletions of edges and decrease of edge weights. In order to do this, we can add a ghost graph, $G_{i}^{\prime}$, such that $\left(G_{i}^{\prime}-G_{i-1}\right)$ contains all the edges added or with increased weights and $\left(G_{i}-G_{i}^{\prime}\right)$ contains all the edges deleted or with decreased weights. The sum of these two closeness difference matrices gives the closeness difference matrix from $G_{i-1}$ to $G_{i}$.

\section{Spotting Significant Subgraphs}

With the closeness difference matrix $\Pi_{i}^{l}$ at time $t_{i}$ and the vertex importance score vector $V I$, we now explain how to expand those vertices of high importance scores to obtain significant changing subgraphs. As mentioned, a changing subgraph is significant if the vertex closeness in the subgraph changes a lot. In our experiments, we find that the vertex importance scores follow the power law distribution. Therefore, instead of defining an absolute threshold for the score, we use the value $\xi$ as the threshold such that more than $80 \%$ of the scores are smaller than it. Apparently, significant changing subgraphs should contain all the important vertices (i.e., those with high importance scores) and 
Table 2. Dataset Characteristics

\begin{tabular}{|c|c|c|c|}
\hline Datasets & Vertices & Avg. Added Edges & Time Steps \\
\hline \hline DB & 5492 & 1734 & 10 \\
\hline DM & 5574 & 1079 & 10 \\
\hline Enron2001 & 16639 & 320 & 184 \\
\hline Enron2002 & 16639 & 203 & 164 \\
\hline
\end{tabular}

most of the vertices whose closenesses with the important vertices change a lot. We develop an expanding strategy which is similar to the density clustering. The basic idea is to include the vertices whose closeness differences with the important vertices are high.

Let the union graph of $G_{i-1}$ and $G_{i}$ keep the information of connectivity. The algorithm starts from an important vertex $v_{j}$ with the maximum importance score in each loop to generate a significant changing subgraph. First, it includes $v_{j}$ into the subgraph. Next, the algorithm adds all the neighbors of $v_{j}$ into a maximum heap $H$. It then repeatedly includes a vertex $v_{k}$ in the heap to the subgraph as long as $\max \left(\Pi_{i}(\mathcal{I}(V(g)), k)\right)$ is larger than the current bound $\epsilon$, where $\epsilon$ is set to $1 / 10$ of the maximum transition probability of the important vertex lastly included into the subgraph. $\mathcal{I}(V(g))$ is the index of all the vertices in $g$. When $\max \left(\Pi_{i}(\mathcal{I}(V(g)), k)\right)$ is smaller than $\epsilon$, we clear the heap and output the current significant changing subgraph. In the final result set of the significant changing subgraphs, two subgraphs are merged if they are directly connected.

\section{Experiments}

We evaluate both the effectiveness and efficiency of our proposed algorithms.

\subsection{Datasets}

The four real datasets are extracted from the DBLP [1] co-authorship dataset and the Enron email dataset [2]. In the DBLP co-authorship dataset, each author is represented by a vertex and there is an edge between two authors if they co-authored some paper. In the Enron email dataset, each email sender or receiver is considered as a vertex and there are edges between senders and receivers. The first two datasets DB and DM are from the DBLP co-authorship dataset. DB contains the co-authorship information of six major database conferences from 1998 to 2007, including SIGMOD, PODS, VLDB, ICDE, EDBT and ICDT. DM contains the co-authorship information of five major data mining conferences from 1998 to 2007, including KDD, ICDM, PKDD, SDM and PAKDD. The other two datasets Enron2001 and Enron2002 are extracted from the Enron email dataset. Enron2001 contains the email communication information of each day from 2001-07-01 to 2001-1231, while Enron2002 contains the email communication in-

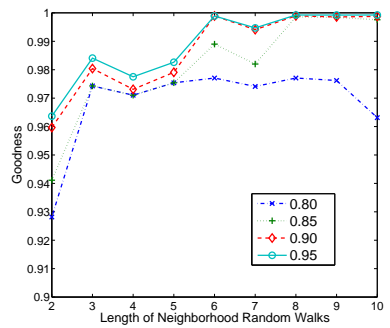

(a) Dataset DB

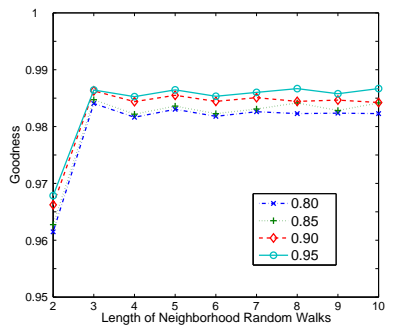

(b) Dataset DM
Figure 2. The Goodness

formation of each day from 2002-01-01 to 2002-7-31. The characteristics of these datasets are summarized in Table 2.

Tong in [10] introduced three aggregation methods: global aggregation, exponential aggregation and sliding window. It is worth noting that our proposed approaches can cooperate with all these three aggregation methods. In this paper, we choose global aggregation to perform our experiments, which aggregates the new edges or edge weights to the adjacency matrix of previous time. Let $\Delta A_{i}$ be the adjacency matrix of the graph at time step $t_{i}$, then $A_{i}=\sum_{t=1}^{i} \Delta A_{t}$. The average number of added edges per time step is presented in Table 2 .

\subsection{Effectiveness}

Let us first introduce our criterion of the significant subgraphs. Let $g_{i}$ denote a significant subgraph found at time $t_{i}$. We evaluate the goodness of significant subgraphs as

$$
\text { Goodness }=\frac{\sum_{v_{j}, v_{k} \in g_{i}} \Delta \Pi_{i}(j, k)}{\sum_{v_{j} \in g_{i}, v_{k} \in G_{i}} \Delta \Pi_{i}(j, k)},
$$

where $\Delta \Pi_{i}(j, k)=\left|\Pi_{i}(j, k)-\Pi_{i-1}(j, k)\right|$, is the closeness difference for $v_{j}$ and $v_{k}$ between $G_{i-1}$ and $G_{i}$. The goodness is essentially the fraction of the closeness differences between $G_{i-1}$ and $G_{i}$ that are captured by significant subgraphs.

We use $c=0.15$ in all experiments. Figures 2(a) and 2(b) present the average goodness for different values of $\xi$, when varying the length of neighborhood random walks $l$ from 2 to 10. For dataset DB, our algorithm captures $92 \%$ changes in vertex closeness, while for dataset DM, our algorithm captures more than $96 \%$. For a higher value of $\xi$ and longer length of $l$, the goodness scores increase.

Two significant subgraphs found are presented as examples in Figures 3(a) and 3(b), which is from the experiments with $l=4$ and $\xi=0.8$. For privacy, we replace author names by abbreviations. The newly added edges are dotted in both subgraphs. Figure 3(a) shows the subgraph from dataset DB. There are originally three communities (dense areas) and the newly added edges make three communities connected, which usually indicates that there is a joint 


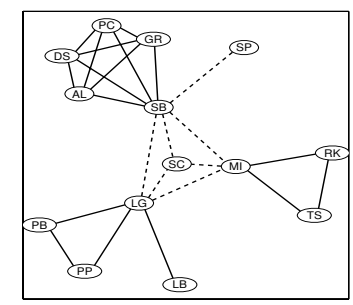

(a) Dataset $\mathbf{D B}$

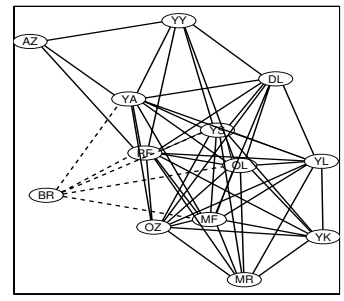

(b) Dataset DM
Figure 3. Significant Subgraphs

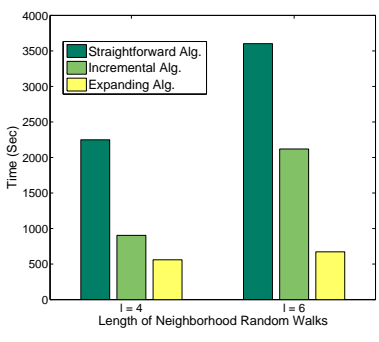

(a) Dataset Enron2001

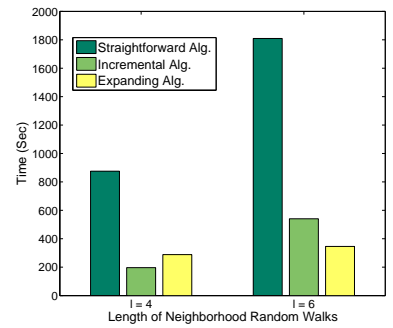

(b) Dataset Enron2002

Figure 4. Overall Running Time

research work involving multiple research groups. Apparently, only the subgraph consisting of the added edges cannot provide this information. There are other vertices connecting to some of the vertices in three communities, and these vertices are not included in the significant subgraph because the difference of the vertex closeness between them and the vertex importance scores are small. In Figure 3(b), the researcher $B R$ co-authored papers with researchers in a very dense community. Researchers in the same research group tend to co-author a lot and form a very dense community. Therefore, it is obvious that $B R$ should be a new member to some research group.

\subsection{Efficiency}

We perform our efficiency testing on datasets Enron2001 and Enron2002. Figures 4(a) and 4(b) show the overall running time for the three algorithms: the straightforward algorithm, the incremental algorithm to compute the vertex importance scores, as well as the expanding algorithm to generate the significant subgraphs. Each figure shows two groups of running time for $l=4$ and $l=6$.

Figure 5(a) presents the average running time of the straightforward algorithm versus the average number of edges in the graphs at each time spot, where we can see that the running time of the straightforward algorithm is proportional to the total number of edges in the graph at current time spot. The average running time of the incremental algorithm versus the average number of the newly added edges in the graphs is shown in Figure 5(b). The running time of the incremental algorithm is proportional to the

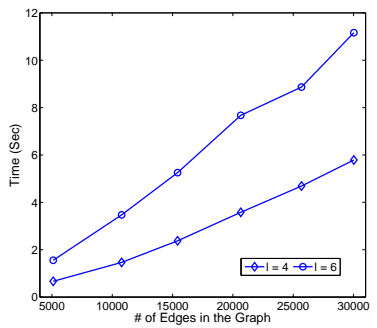

(a) The Straightforward Algorithm

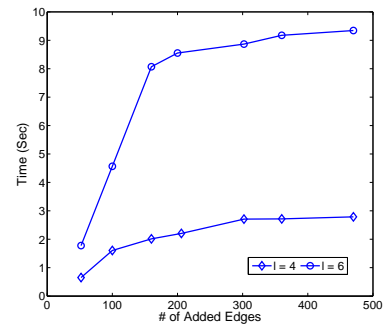

(b) The Incremental Algorithm
Figure 5. Average Running Time

total number of edges added. This explains why the incremental algorithm is faster.

\section{Conclusions}

We study the challenging problem of spotting significant changing subgraphs in evolving graphs in this paper. We propose to use the neighborhood random walk distance to measure the vertex closeness, as well as a novel incremental algorithm for fast computation. The significant subgraphs are generated based on the vertex importance score. Experimental results show that our approach can solve this problem effectively and efficiently.

\section{Acknowledgment}

This work was supported by a grant of RGC, Hong Kong SAR, China (No. CUHK419008).

\section{References}

[1] http://www.informatik.uni-trier.de/ ley/db/.

[2] http://www.cs.cmu.edu/ enron/.

[3] H. Bunke, P. J. Dickinson, M. Kraetzl, and W. D. Wallis. A Graph-Theoretic Approach to Enterprise Network Dynamics. Birkhauser, 2006.

[4] C. Faloutsos, K. S. McCurley, and A. Tomkins. Fast discovery of connection subgraphs. In KDD. ACM, 2004.

[5] G. Jeh and J. Widom. Simrank: a measure of structuralcontext similarity. In KDD. ACM, 2002.

[6] G. Jeh and J. Widom. Scaling personalized web search. In $W W W$. ACM, 2003.

[7] J.-Y. Pan, H.-J. Yang, C. Faloutsos, and P. Duygulu. Automatic multimedia cross-modal correlation discovery. In KDD. ACM, 2004.

[8] B. E. Shakhnovich and J. M. Harvey. Quantifying structurefunction uncertainty: A graph theoretical exploration into the origins and limitations of protein annotation. Journal of Molecular Biology, 4(337), 2004.

[9] H. Tong, C. Faloutsos, and Y. Koren. Fast direction-aware proximity for graph mining. In KDD. ACM, 2007.

[10] H. Tong, S. Papadimitriou, P. S. Yu, and C. Faloutsos. Proximity tracking on time-evolving bipartite graphs. In $S D M$, 2008. 